\title{
ON HYDRODYNAMICS IN THE PRESENSE OF STRONG EXTERNAL POTENTIAL FIELD
}

\author{
A.I. Sokolovsky ${ }^{1 *}$, S.A. Sokolovsky ${ }^{2}$ \\ ${ }^{1}$ Oles Honchar Dnipro National University, Dnipro, Ukraine \\ ${ }^{2}$ Prydniprovska State Academy of Civil Engineering and Architecture, Dnipro, Ukraine \\ *e-mail: alexander.i.sokolovsky@gmail.com
}

On the base of the Boltzmann kinetic equation, hydrodynamics of a dilute gas in the presence of the strong external potential field is investigated. First of all, a gravitational field is meant, because the consistent development of hydrodynamics in this environment is of great practical importance. In the present paper it is assumed that it is possible to neglect the influence of the field on the particle collisions. The study is based on the Chapman-Enskog method in a Bogolyubov's formulation, which uses the idea of the functional hypothesis. Consideration is limited to steady gas states, which are subjected to a simpler experimental study. Chemical potential $\mu_{0}$ of the gas at the point where the external field has zero value

and its temperature $T$ are selected as the reduced description parameters of the system. In equilibrium, in the presence of the field, these values do not depend on the coordinates. It is assumed that in the hydrodynamic states $T$ and $\mu_{0}$ are weakly dependent on the coordinates and therefore their gradients, considered on the scale of the free path length of the gas, are small. The kinetic equation, accounting for the functional hypothesis, gives an integro-differential equation for a gas distribution function at the hydrodynamic stage of evolution. This equation is solved in perturbation theory in gradients of $T$ and $\mu_{0}$. The main approximation is analyzed for possibility of the system to be in a local equilibrium by means of comparing it with an equilibrium distribution function. Next, the distribution function is calculated in the first approximation in gradients and it is expressed in terms of solutions $A_{p}, B_{p}$ of some first kind integral Fredholm equations. An approach to the approximate solution of these equations is discussed. The found distribution function is used to calculate the fluxes of the number of gas particles and their energy in the first order in gradients $T$ and $\mu_{0}$. Kinetic coefficients, which describe the structure of these fluxes, are introduced. Matrix elements of the operator of the linearized collision integral (integral brackets) are used for their research. It is a question of validity of the principle of symmetry of kinetic coefficients and definition of their signs.

Keywords: Boltzmann kinetic equation, hydrodynamic equation, strong external potential field, functional hypothesis, parameters of reduced description, steady states, kinetic coefficients.

Received 22.06.2021; Received in revised form 25.07.2021; Accepted 15.08.2021

\section{Introduction}

The study of hydrodynamic processes in various systems is a modern direction in the theory of nonequilibrium processes [1]. Particular attention is paid to the study of hydrodynamic states of systems whose equilibrium state is spatially inhomogeneous. Such systems include both systems in the external field, which causes inhomogeneity, and systems with a broken symmetry. The most important examples are the hydrodynamics of a normal fluid in a gravitational field [2], the hydrodynamics of a plasma in an electric field [3], the hydrodynamics of a superfluid Bose fluid (see, for example, [4]), and the hydrodynamics of crystalline bodies (see, in particular, [5]). The developed approaches are reduced to the search for quantities that are close to the values of standard hydrodynamics, but for spatially inhomogeneous considered system do not depend on the coordinates. In the hydrodynamic state of a spatially inhomogeneous system, it is assumed that these quantities are weakly dependent on the coordinates, i.e., their gradients are small.

The basis of fluid hydrodynamics to some extent is its thermodynamics [6]. It should be noted that there are fundamental issues related to the justification of the thermodynamics of systems in the external potential field $U(x)$. The basic statement [3] on the independence of coordinates for an arbitrary one-component fluid of quantity $\mu(n(x), T)+U(x)$, where $\mu(n, T)$ is the chemical potential of the fluid as a function of the density of its particle number $n(x)$ 
and temperature $T$, requires further study.

In this work, the hydrodynamic states of a one-component gas in the presence of an external potential field $U(x)$ are investigated. The consideration is based on the Boltzmann kinetic equation, which has usual form

$$
\frac{\partial \mathrm{f}_{p}(x, t)}{\partial t}=-\frac{\partial \varepsilon_{p}}{\partial p_{l}} \frac{\partial \mathrm{f}_{p}(x, t)}{\partial x_{l}}+\frac{\partial U(x)}{\partial x_{l}} \frac{\partial \mathrm{f}_{p}(x, t)}{\partial p_{l}}+I_{p}(\mathrm{f}(x, t))
$$

$\left(\varepsilon_{p}=p^{2} / 2 m\right)$. In this case, the force acting on each particle $F_{l}=-\partial U / \partial x_{l}$ is not considered small, i.e. the field $U$ is not considered weak. At the same time, the influence of the field on the pair collisions of the gas particles is neglected. The collision integral of the kinetic equation can be written in the form

$$
\begin{gathered}
I_{p}(\mathrm{f}) \equiv \int d^{3} p_{1} d^{3} p_{2} d^{3} p_{3} d^{3} p_{4} \Phi\left(p_{1} p_{2}, p_{3} p_{4}\right) \times \\
\times\left\{\delta\left(p-p_{1}\right)+\delta\left(p-p_{2}\right)-\delta\left(p-p_{3}\right)-\delta\left(p-p_{4}\right)\right\} \times \\
\times \delta\left(p_{1}+p_{2}-p_{3}-p_{4}\right) \delta\left(\varepsilon_{1}+\varepsilon_{2}-\varepsilon_{3}-\varepsilon_{4}\right)\left\{\mathrm{f}_{3} \mathrm{f}_{4}-\mathrm{f}_{1} \mathrm{f}_{2}\right\},
\end{gathered}
$$

which emphasizes the conservation in collisions of the number of particles, their momentum and energy (to simplify recording $\varepsilon_{i} \equiv \varepsilon_{p_{i}}, \mathrm{f}_{i}=\mathrm{f}_{p_{i}}$ ) and allows to easily check the relations

$$
\int d^{3} p I_{p}(\mathrm{f})=0, \quad \int d^{3} p I_{p}(\mathrm{f}) p_{l}=0, \quad \int d^{3} p I_{p}(\mathrm{f}) \varepsilon_{p}=0 .
$$

The densities of the number of particles $n(x)$, momentum $\pi_{l}(x)$ and energy $\varepsilon(x)$ are determined by conventional formulas

$$
n(x, t)=\int d \tau_{p} \mathrm{f}_{p}(x, t), \quad \pi_{l}(x, t)=\int d \tau_{p} \mathrm{f}_{p}(x, t) p_{l}, \quad \varepsilon(x, t)=\int d \tau_{p} \mathrm{f}_{p}(x, t) \varepsilon_{p}
$$

(for reasons of dimensionality, the element of integration is chosen in the form $d \tau_{p} \equiv d^{3} p / h^{3}$ where $h$ is Planck's constant). The basis of the equations of hydrodynamics is the laws of conservation in differential form, which follow from the kinetic equation and in the presence of an external field have the form

$$
\frac{\partial n}{\partial t}=-\frac{\partial i_{l}}{\partial x_{l}}, \quad \frac{\partial \pi_{n}}{\partial t}=-\frac{\partial t_{n l}}{\partial x_{l}}-n \frac{\partial U}{\partial x_{n}}, \quad \frac{\partial \varepsilon}{\partial t}=-\frac{\partial q_{l}}{\partial x_{l}}-\frac{\pi_{l}}{m} \frac{\partial U}{\partial x_{l}} .
$$

where the flux densities of the number of particles $i_{l}$, momentum $t_{n l}$ and energy $q_{l}$

$$
\begin{gathered}
i_{l}(x, t)=\pi_{l}(x, t) / m, \quad t_{n l}(x, t)=\int d \tau_{p} \mathrm{f}_{p}(x, t) p_{n} \frac{\partial \varepsilon_{p}}{\partial p_{l}}, \\
q_{l}(x, t)=\int d \tau_{p} \mathrm{f}_{p}(x, t) \varepsilon_{p} \frac{\partial \varepsilon_{p}}{\partial p_{l}}
\end{gathered}
$$

are introduced. The problem of constructing the equations of standard hydrodynamics is to find expressions for the densities of momentum $t_{n l}$ and energy $q_{l}$ fluxes as functions of densities $n, \pi_{l}, \varepsilon$. 
Note that a similar problem was considered by Lorentz and with the time by Landau for plasma in the Lorentz model [3]. Their work was based on the kinetic equation in the approximation of the relaxation time, taking into account the external electric field and to some extent the effects of long-range. Our paper is devoted to the study of a gas primarily in an external gravitational field, the interaction between the particles of which is shortrange one and the dynamics of which is described by the Boltzmann kinetic equation.

The work is structured as follows. Section 2 formulates an approach to the construction of gas hydrodynamics in the presence of a strong potential external field. Section 3 derives general formulas for gas kinetic coefficients and discusses their properties.

\section{Construction of gas hydrodynamics in the presence of an external field}

The equilibrium gas distribution function in the presence of an external potential field $U(x)$ has the form

$$
\begin{gathered}
\mathrm{f}_{p}^{e q}(x)=h^{3} n^{e q}(x) w_{p-m v}, \quad n^{e q}(x)=n_{0} e^{-\frac{U(x)}{T}}, \quad w_{p}=\frac{1}{(2 \pi m T)^{3 / 2}} e^{-\frac{\varepsilon_{p}}{T}} \\
\int d \tau_{p} \mathrm{f}_{p}^{e q}(x)=n^{e q}(x),
\end{gathered}
$$

where $n^{e q}(x)$ is the Boltzmann distribution for the density of the number of particles, $w_{p}$ is the Maxwell distribution, $v_{l}$ is gas velocity, $n_{0}$ is gas density at the point where the field is zero. This function satisfies kinetic equation (1) with quantities $n_{0}, T, v_{l}$ independent on coordinates and time.

The equilibrium distribution function $\mathrm{f}_{p}^{e q}(x)$ at rest of the gas can be written as the Maxwell-Boltzmann distribution

$$
\begin{gathered}
\mathrm{f}_{p}^{e q}(x)=e^{\frac{\mu_{0}-\left[\varepsilon_{p}+U(x)\right]}{T}} . \\
\mu_{0} \equiv \mu\left(n_{0}, T\right), \quad \mu(n, T)=T \ln \frac{n h^{3}}{(2 \pi m T)^{3 / 2}},
\end{gathered}
$$

where the chemical potential of the classical ideal gas is introduced. The value

$$
\mu(n(x), T)+U(x)=\mu_{0}
$$

and does not depend on coordinates.

In this paper, we limit ourselves to the study of steady-state gas states, which are the most important from the point of view of the experiment. In the steady state of the system, the parameters that describe its state do not depend on time, but it has fluxes of particles, momentum and energy. Consider hydrodynamic states, which are described by the parameters $\mu_{0}(x), T(x)$ and assuming that the gas is at rest. The gradients of these parameters are considered to be small, because in equilibrium they do not depend on the coordinates. As usual the next estimates

$$
\frac{\partial^{s} \mu_{0}(x)}{\partial x_{l_{1}} . \partial x_{l_{s}}} \sim g^{s}, \quad \frac{\partial^{s} T(x)}{\partial x_{l_{1}} . \partial x_{l_{s}}} \sim g^{s}
$$


are used. Small parameter of the theory $g$ is introduced on the basis of estimates of the type

$$
\frac{\partial^{s} T(x)}{\partial x_{l_{1}} . \partial x_{l_{s}}} \sim \frac{T}{L^{s}}, \quad g \equiv \frac{l}{L}
$$

where $l$ is the free path length, $L$ is the characteristic size of inhomogeneities in the system, $T$ is the characteristic temperature value.

The consideration of the paper is based on the Chapman-Enskog method [6] in Bogolyubov's formulation (see [7]), the basis of which is his idea of the functional hypothesis

$$
\mathrm{f}_{p}(x, t) \underset{t>>\tau_{0}}{\longrightarrow} \mathrm{f}_{p}\left(x, \mu_{0}(t), T(t)\right)
$$

( $\tau_{0}$ is characteristic time of the order of free path time). Nonequilibrium distribution function $\mathrm{f}_{p}\left(x, \mu_{0}, T\right)$ is sought from the kinetic equation in the form of an expansion in the gradients of parameters $\mu_{0}(x)$ and $T(x)$

$$
\mathrm{f}_{p}\left(x, \mu_{0}, T\right)=\mathrm{f}_{p}^{(0)}(x)+\mathrm{f}_{p}^{(1)}(x)+O\left(g^{2}\right)
$$

In zero order approximation, the distribution function is given by the local MaxwellBoltzmann distribution

$$
\mathrm{f}_{p}^{(0)}(x)=\omega_{p}(x), \quad \omega_{p}(x) \equiv e^{\frac{\mu_{0}(x)-\left[\varepsilon_{p}+U(x)\right]}{T(x)}}
$$

because

$$
\left(-\frac{\partial \varepsilon_{p}}{\partial p_{l}} \frac{\partial \omega_{p}}{\partial x_{l}}+\frac{\partial U}{\partial x_{l}} \frac{\partial \omega_{p}}{\partial p_{l}}\right)^{(0)}=0, \quad I_{p}(\omega)=0 .
$$

When analyzing the first in the gradient approximation, it is taken into account that

$$
-\frac{\partial \varepsilon_{p}}{\partial p_{l}} \frac{\partial \mathrm{f}_{p}^{(0)}}{\partial x_{l}}+\frac{\partial U}{\partial x_{l}} \frac{\partial \mathrm{f}_{p}^{(0)}}{\partial p_{l}}=-\frac{p_{l}}{m}\left\{\frac{1}{T} \frac{\partial \mu_{0}}{\partial x_{l}}-\frac{\mu_{0}-\left[\varepsilon_{p}+U\right]}{T^{2}} \frac{\partial T}{\partial x_{l}}\right\} \omega_{p}
$$

and

$$
\begin{gathered}
I_{p}(\mathrm{f})=I_{p}\left(\mathrm{f}^{(0)}+\delta \mathrm{f}\right)=I_{p}\left(\mathrm{f}^{(0)}\right)+\left.\int d^{3} p^{\prime} \frac{\delta I_{p}(\mathrm{f})}{\delta \mathrm{f}_{p^{\prime}}}\right|_{f=f^{(0)}} \delta \mathrm{f}_{p^{\prime}}+O\left(\delta \mathrm{f}^{2}\right) \equiv \\
\equiv \int d^{3} p^{\prime} M_{p p^{\prime}} \delta \mathrm{f}_{p^{\prime}}+O\left(\delta \mathrm{f}^{2}\right) .
\end{gathered}
$$

The integral equation for the first-order contribution to the distribution function has the form

$$
0=-\frac{p_{l}}{m}\left\{\frac{1}{T} \frac{\partial \mu_{0}}{\partial x_{l}}-\frac{\mu_{0}-\varepsilon_{p}-U(x)}{T^{2}} \frac{\partial T}{\partial x_{l}}\right\} \omega_{p}+\int d^{3} p^{\prime} M_{p p^{\prime}} \mathrm{f}_{p^{\prime}}^{(1)}
$$

Its solution for reasons of rotational invariance has the structure 


$$
\mathrm{f}_{p}^{(1)}=\omega_{p} h_{p}, \quad h_{p} \equiv A_{p} p_{l} \frac{\partial \mu_{0}}{\partial x_{l}}+B_{p} p_{l} \frac{\partial T}{\partial x_{l}},
$$

where $A_{p}, B_{p}$ are some scalar functions which are solutions of the integral equations

$$
\int d^{3} p^{\prime} K_{p p^{\prime}} A_{p^{\prime}} p_{l}^{\prime}=\frac{1}{m T} p_{l}, \quad \int d^{3} p^{\prime} K_{p p^{\prime}} B_{p^{\prime}} p_{l}^{\prime}=\frac{\varepsilon_{p}-\mu_{0}}{m T^{2}} p_{l} .
$$

The kernel of these integral equations is determined by the collision integral with formulas

$$
\begin{gathered}
\omega_{p} K_{p p^{\prime}}=-M_{p p^{\prime}} \omega_{p^{\prime}}, \\
M_{p p^{\prime}}=\int d^{3} p_{1} d^{3} p_{2} d^{3} p_{3} d^{3} p_{4} \Phi\left(p_{1} p_{2}, p_{3} p_{4}\right) \times \\
\times\left\{\delta\left(p-p_{1}\right)+\delta\left(p-p_{2}\right)-\delta\left(p-p_{3}\right)-\delta\left(p-p_{4}\right)\right\} \times \\
\times \delta\left(p_{1}+p_{2}-p_{3}-p_{4}\right) \delta\left(\varepsilon_{1}+\varepsilon_{2}-\varepsilon_{3}-\varepsilon_{4}\right) \times \\
\times\left\{\delta\left(p^{\prime}-p_{3}\right) \omega_{4}+\delta\left(p^{\prime}-p_{4}\right) \omega_{3}-\delta\left(p^{\prime}-p_{1}\right) \omega_{2}-\delta\left(p^{\prime}-p_{2}\right) \omega_{1}\right\}, \\
K_{p p^{\prime}}=\omega_{p} \int d^{3} p_{1} d^{3} p_{2} d^{3} p_{3} d^{3} p_{4} \Phi\left(p_{1} p_{2}, p_{3} p_{4}\right) \times \\
\times\left\{\delta\left(p-p_{1}\right) \omega_{2}+\delta\left(p-p_{2}\right) \omega_{1}-\delta\left(p-p_{3}\right) \omega_{4}-\delta\left(p-p_{4}\right) \omega_{3}\right\} \times \\
\times \delta\left(p_{1}+p_{2}-p_{3}-p_{4}\right) \delta\left(\varepsilon_{1}+\varepsilon_{2}-\varepsilon_{3}-\varepsilon_{4}\right) \times \\
\times\left\{\delta\left(p^{\prime}-p_{1}\right)+\delta\left(p^{\prime}-p_{2}\right)-\delta\left(p^{\prime}-p_{3}\right)-\delta\left(p^{\prime}-p_{4}\right)\right\}
\end{gathered}
$$

$\left(\omega_{i} \equiv \omega_{p_{i}}\right)$. Integral equations (20) do not contain any small parameter that can be used to approximately solve them. An approximate solution can be found by the method of truncated expansion in the Sonine polynomials (a special case of the Galerkin method) (see [3]).

\section{Kinetic coefficients of the gas in the presence of external field}

The found distribution function (19) allows calculating by formulas (4), (6) the fluxes of the number and energy of particles

$$
i_{l}^{(1)}=\int d \tau_{p} \frac{\partial \varepsilon_{p}}{\partial p_{l}} \mathrm{f}_{p}^{(1)}, \quad q_{l}^{(1)}=\int d \tau_{p} \frac{\partial \varepsilon_{p}}{\partial p_{l}} \varepsilon_{p} \mathrm{f}_{p}^{(1)} .
$$

With the considerations of rotational invariance, this gives

$$
i_{l}^{(1)}=-a \frac{\partial \mu_{0}}{\partial x_{l}}-b \frac{\partial T}{\partial x_{l}}, q_{l}^{(1)}=-c \frac{\partial \mu_{0}}{\partial x_{l}}-d \frac{\partial T}{\partial x_{l}}
$$

where kinetic coefficients

$$
a \equiv \frac{2}{3}\left\langle\varepsilon_{p} A_{p}\right\rangle, \quad b \equiv \frac{2}{3}\left\langle\varepsilon_{p} B_{p}\right\rangle, \quad c \equiv \frac{2}{3}\left\langle\varepsilon_{p}^{2} A_{p}\right\rangle d \equiv \frac{2}{3}\left\langle\varepsilon_{p}^{2} B_{p}\right\rangle .
$$

are introduced. 
One can analyze the properties of the kinetic coefficients, expressing them through the bilinear form $\left\{a_{p}, b_{p}\right\}$

$$
\begin{gathered}
\left\{a_{p}, b_{p}\right\} \equiv \int d^{3} p d^{3} p^{\prime} \omega_{p} a_{p} K_{p p^{\prime}} b_{p^{\prime}}=, \\
=\int d^{3} p_{1} d^{3} p_{2} d^{3} p_{3} d^{3} p_{4} \Phi\left(p_{1} p_{2}, p_{3} p_{4}\right) \omega_{p_{1}} \omega_{p_{2}} \times \\
\times \delta\left(p_{1}+p_{2}-p_{3}-p_{4}\right) \delta\left(\varepsilon_{p_{1}}+\varepsilon_{p_{2}}-\varepsilon_{p_{3}}-\varepsilon_{p_{4}}\right) \times \\
\times\left\{a_{p_{1}}+a_{p_{2}}-a_{p_{3}}-a_{p_{4}}\right\}\left\{b_{p_{1}}+b_{p_{2}}-b_{p_{3}}-b_{p_{4}}\right\} .
\end{gathered}
$$

The bilinear form $\left\{a_{p}, b_{p}\right\}$, which is also called the integral bracket [6], has useful properties for arbitrary functions $a_{p}, b_{p}$.

$$
\begin{gathered}
\left\{a_{p}, b_{p}\right\}=\left\{b_{p}, a_{p}\right\}, \quad\left\{a_{p}, a_{p}\right\} \geq 0, \\
\left\{a_{p}, a_{p}\right\}\left\{b_{p}, b_{p}\right\} \geq\left\{a_{p}, b_{p}\right\}^{2} .
\end{gathered}
$$

Integral equations (20) for functions $A_{p}, B_{p}$, and definitions of kinetic coefficients (24) give identities

$$
\begin{aligned}
& (A, A) \equiv\left\{A_{p} p_{l}, A_{p} p_{l}\right\}=\frac{3}{T} a, \quad(B, B) \equiv\left\{B_{p} p_{l}, B_{p} p_{l}\right\}=\frac{3}{T^{2}}\left(d-\mu_{0} b\right) \\
& (B, A) \equiv\left\{B_{p} p_{l}, A_{p} p_{l}\right\}=\frac{3}{T} b, \quad(A, B) \equiv\left\{A_{p} p_{l}, B_{p} p_{l}\right\}=\frac{3}{T^{2}}\left(c-\mu_{0} a\right),
\end{aligned}
$$

As a result, the formulas for fluxes (23) and the corresponding kinetic coefficients take the form

$$
\begin{gathered}
i_{l}^{(1)}=-a \frac{\partial \mu_{0}}{\partial x_{l}}-b \frac{\partial T}{\partial x_{l}}, \quad q_{l}^{(1)}=\mu_{0} i_{l}^{(1)}-T b \frac{\partial \mu_{0}}{\partial x_{l}}-k \frac{\partial T}{\partial x_{l}}, \\
a=\frac{T}{3}(A, A)>0, \quad b=\frac{T}{3}(A, B), \quad k \equiv \frac{T^{2}}{3}(B, B)>0 .
\end{gathered}
$$

The energy flux includes the same kinetic coefficient $b$ as the particle flux. This is a manifestation of the Onsager principle of symmetry of kinetic coefficients. The first term in the energy flux should be considered as convective transfer, i.e. energy transfer together with particle transfer. The thermal conductivity $\kappa$ should be defined by the formula

$$
q_{l}^{(1)}=\left(\mu_{0}+\frac{b T}{a}\right) i_{l}^{(1)}-\kappa \frac{\partial T}{\partial x_{l}}
$$

that is, by the transfer of energy in the absence of the flux of particles that gives

$$
\kappa=\frac{T^{2}}{3} \frac{(A, A)(B, B)-(A, B)^{2}}{(A, A)}>0 .
$$


Thus, the expressions for all kinetic coefficients of the system are obtained and their general properties are clarified. Approximate solutions of integral equation (20) for $A_{p}$, $B_{p}$ and calculation of the kinetic coefficients will be given in a subsequent paper.

\section{Conclusions}

The hydrodynamics of steady states of rarefied gas in the presence of a strong potential external field $U(x)$ under the condition of absence of macroscopic motion in the system is developed in the work. In the gas, the force $F_{l}=-\partial U / \partial x_{l}$ acts on its particle that is not considered small, but its effect on the collision of particles is neglected. Generalization for the case of non-steady states of a moving gas will be carried out in a subsequent paper. This can be done quite simply, because, despite the finite magnitude of the force, the contributions of zero order in gradients in the general equations of hydrodynamics (5) are absent. This can be seen from formula (16) when replacing the distribution function $\omega_{p}$ in it by the function $\omega_{p-m v}$, where $v_{l}$ is the macroscopic velocity of the gas.

The present paper is based on the Chapman-Enskog method in Bogolyubov's formulation, which is based on his idea of the functional hypothesis. As part of the construction of hydrodynamics based on the Boltzmann kinetic equation, the system is considered largely as an ideal gas. In particular, this can be seen from the definitions of energy density (4) and flux densities of energy and momentum (6). This is also seen from expression (7) for the equilibrium distribution function $\mathrm{f}_{p}^{e q}(x)$, which is exact solution of the kinetic equation and contains the Boltzmann distribution for the particle density in the presence of a potential field. Expression (8) for $\mathrm{f}_{p}^{e q}(x)$ allows to define as hydrodynamic states of the gas such ones in which chemical potential $\mu_{0}$ and temperature $T$ weakly depend on coordinates and therefore are parameters of the reduced description $\left(\mu_{0}\right.$ is chemical potential of the gas at a point where the field potential $U(x)$ is equal to zero).

It is further proved that the localized equilibrium distribution function $\omega_{p}(x)$ with (14) is a contribution to the nonequilibrium distribution function $\mathrm{f}_{p}\left(x, \mu_{0}, T\right)$ of zero order in gradients of the parameters of the reduced description $\mu_{0}(x)$ and $T(x)$. This means that the gas in the presence of a potential external field can be in a local equilibrium and is allowed to calculate the contribution $\mathrm{f}_{p}^{(1)}\left(x, \mu_{0}, T\right)$ of the first order in gradients of $\mu_{0}$ i $T$ to $\mathrm{f}_{p}\left(x, \mu_{0}, T\right)$. This contribution is determined by two scalar functions $A_{p}$ and $B_{p}$, which are solutions of Fredholm integral equations of the first kind with the positively defined kernel. These equations do not contain any small parameter and cannot be solved in a perturbation theory. Their approximate solution will be investigated in a subsequent paper on the basis of the method of truncated expansion in orthogonal Sonine polynomials (a special case of the Galerkin method) (see, for example, [3]).

Finally kinetic coefficients of the gas are calculated in terms of functions $A_{p}$ and $B_{p}$, written through the matrix elements of the operator $K_{p p^{\prime}}$ of linearized collision integral (integral brackets). This allowed us to note the implementation of the Onsager principle of symmetry of kinetic coefficients and determine their signs. 


\section{References}

1. Colangeli, M. From Kinetic Models to Hydrodynamics: Some Novel Results / M. Colangeli - New York: Springer, 2013. - 104 p.

2. Landau, L.D. Fluid Mechanics // L.D. Landau, E.M. Lifshiz. - Oxford: Pergamon Press, 1982. - 539 p.

3. Lifshitz, E.M. Physical Kinetics // E.M. Lifshiz, L.P. Pitaevskii. - Oxford: Pergamon Press, 1982. - 452 p.

4. Peletminskii, S.V. Hydrodynamics of a superfluid Bose liquid with allowance for dissipative processes in a model with weak interaction / S.V. Peletminskii, A.I. Sokolovsky, V.S. Schelokov // Theoretical and Mathematical Physics. - 1978. -V.34, No.1. - P.51-61).

5. Lavrinenko, N.M. Metod integralnogo uravneniya v teorii slabo neravnovesnykh sostoyanij tverdogo tela / N.M. Lavrinenko, A.I. Sokolovskij // UFZh. - 1985. - T.30, №4. - C.589-597.

6. Jou, D. Extended Irreversible Thermodynamics / D. Jou, J. Casas-Vázques, G. Lebon. - N.Y.: Springer, 2010. -483 p.

7. Ferziger, J.H. Mathematical Theory of Transport Processes in Gases / J.H. Ferziger, H.G. Kaper. - Amsterdam: North-Holland, 1972. - 554 p.

8. Akhiezer, A.I. Methods of Statistical Physics/ A.I. Akhiezer, S.V. Peletminskii. Amsterdam: Elsevier. - 2013. - 446 p. 